\title{
Fluorescence Imaging of Actin Fine Structure in Tumor Tissues Using SiR-Actin Staining
}

\author{
NATALIA V. KLEMENTIEVA ${ }^{1}$, LUDMILA B. SNOPOVA ${ }^{1}$, NATALIA N. PRODANETS ${ }^{1}$, \\ OLGA E. FURMAN ${ }^{2}$, VARVARA V. DUDENKOVA ${ }^{1,2}$, ELENA V. ZAGAYNOVA ${ }^{1}$, \\ KONSTANTIN A. LUKYANOV ${ }^{1,3}$ and ALEXANDER S. MISHIN ${ }^{1,3}$ \\ ${ }^{1}$ Nizhny Novgorod State Medical Academy, Nizhny Novgorod, Russian Federation; \\ ${ }^{2}$ Lobachevsky State University of Nizhny Novgorod, Nizhny Novgorod, Russian Federation; \\ ${ }^{3}$ Shemyakin-Ovchinnikov Institute of Bioorganic Chemistry, Moscow, Russian Federation
}

\begin{abstract}
Background: The rearrangement of actin cytoskeleton is being increasingly considered a marker of cancer cell activity, but the fine structure and remodeling of microfilaments within tumor tissue still remains unclear. Materials and Methods: We used the recently introduced silicon-rhodamine (SiR)-actin dye to visualize endogenous actin within tissues by confocal or total internal reflection fluorescence microscopy. We established imaging conditions for robust blinking of SiR-actin, which makes this dye applicable for super-resolution localization microscopy, as well as for an efficient background elimination. Results: We studied tumor tissue samples in two mouse models at high resolution and revealed a complex network of thick curved bundles of actin in cancer cells in tumors. This actin pattern differed strongly from that in cancer cells in vitro and in normal tissues. Conclusion: Localization microscopy with SiR-actin provides an efficient way to visualize fine actin structure in tumor tissues. It is potentially applicable to a variety of biological and clinical samples.
\end{abstract}

Actin, being an essential building block of the cytoskeleton, is responsible for many cellular functions, from cell motility, endocytosis, and growth to cytokinesis and even regulation of transcription $(1,2)$. It is one of the most abundant and highly conserved proteins of eukaryotic cells. Polymerization and disassembly of cellular actin occur all the time. Globular G-actin organizes into flexible filamentous F-actin, forming

Correspondence to: Alexander S. Mishin, Ph.D., Institute of Bioorganic Chemistry, Miklukho-Maklaya 16/10, 117997 Moscow, Russian Federation. Tel: +7 4997248122, Fax: +7 4953307056,e-mail: mishin@ibch.ru

Key Words: Actin fine structure, tumor tissue, fluorescence imaging, super-resolution microscopy, SiR-actin. specialized cellular structures such as lamellipodia, filopodia, and podosomes (3-5).

Although the actin cytoskeleton is essential for normal cell functioning, it can also be involved in tumor progression. Some properties inherent to cancer cells, such as altered stiffness, motility and invasiveness, are intrinsically linked to actin filament bundling (6). The actin cytoskeleton is thought to initiate formation of various protrusions (7), or be disrupted in the course of the cell transformation (8). Different actin isoforms can either promote or suppress tumorigenesis (9). However, it is still unclear in exactly what manner adhesive and mechanical properties of cancer cells are associated with their invasive potential (10).

Different tumors vary in their malignancy and, consequently, cancer cells capable of metastasis should display unique properties (11). More aggressive cancer cells are expected to rely heavily on changes in their actin cytoskeleton, which may be manifested in epithelialmesenchymal transition, including stress fiber formation and cell elongation (12). The ability of cancer cells to change cell shape and plasticity due to actin cytoskeleton rearrangement is an important regulatory mechanism of the metastatic cascade (13).

It is understood that the ultrastructure of F-actin and its reorganization in cancer cells should be visualized very accurately in order to be able to precisely define microfilament architecture (14). Nowadays, emerging superresolution localization-based microscopy techniques, offering significant improvement in resolution, down to $10-30 \mathrm{~nm}$ $(15,16)$, provide the means for in-depth investigation of complex actin structures $(17,18)$.

The understanding of how a number of $2 \mathrm{D}$ or $3 \mathrm{D}$ cell culture models relate to the true tumor environment remains a challenge (19). Despite super-resolution microscopy being recently applied for cytoskeleton visualization in hippocampal tissue samples (20), the finest details of actin 
in tumors are poorly characterized. To the best of our knowledge, there are few data on actin filament structure within tumor tissue $(21,22)$.

In the current study, we examined the structure of the actin cytoskeleton at high resolution in tissue samples of animal tumor models. We applied staining with recently introduced fluorogenic silicon-rhodamine (SiR)-actin dye (23) in conditions favourable for dye blinking, followed by superresolution image reconstruction. As a result, we demonstrated existence of intricate network of thick actin bundles in tumor tissue cells that was in a contrast to normal cells of the same origin lacking such actin bundling.

\section{Materials and Methods}

Cell culture. Human cervical carcinoma (HeLa), mouse colonic carcinoma (CT26) and Lewis lung carcinoma (LLC1) cell lines were kindly provided by Shemyakin-Ovchinnikov Institute of Bioorganic Chemistry of the Russian Academy of Sciences, Herzen Moscow Oncology Research Institute and Bakh Institute of Biochemistry (Moscow, Russia), respectively. HeLa and CT26 cells were cultured in Dulbecco's modified Eagle's medium (DMEM) containing $10 \%$ fetal bovine serum (FBS; GE Healthcare Life Sciences, Logan, UT, USA), $50 \mathrm{U} / \mathrm{ml}$ penicillin, $50 \mu \mathrm{g} / \mathrm{ml}$ streptomycin and 4 мM L-glutamine (Paneco, Moscow, Russia), in an atmosphere of humidified air-5\% $\mathrm{CO}_{2}$ at $37^{\circ} \mathrm{C}$. LLC1 cells were grown in DMEM/F12 medium containing $20 \%$ FBS, $50 \mathrm{U} / \mathrm{ml}$ penicillin, $50 \mu \mathrm{g} / \mathrm{ml}$ streptomycin and $4 \mathrm{MM} \mathrm{L}$-glutamine in the presence of $1 / 3$ conditioned medium. Subculturing was performed every 2-4 days, at $80 \%$ confluency. Before subcutaneous injection into mice, CT26 and LLC1 cells were harvested using trypsinEDTA and washed with phosphate-buffered saline (PBS; Paneco, Moscow, Russia) according to a standard protocol. HeLa cells were used for the validation of SiR-actin-based super-resolution imaging protocol. Live and paraformaldehyde-fixed (PFA) cells were stained with SiR-actin as described previously (23).

Animals and tumor models. Female Balb/c and male C57BL/6 mice were obtained from the vivarium of the Pushchino Branch of the Institute of Bioorganic Chemistry (Moscow Region, Russia). We used mice, 3 to 4 months old, weighing 18-22 g, without any phenotypical or developmental deviations. The animals were housed in the conventional clean facilities. All experiments were performed in accordance with the European Convention for the Protection of Vertebrate Animals used for Experimental and other Scientific Purposes and approved by the Ethics Committee of the Nizhny Novgorod State Medical Academy (Protocol No. 14 of December $10,2013)$. Primary tumors were formed by subcutaneous injection of 500,000 CT26 or LLC1 cancer cells in $100 \mu \mathrm{l}$ PBS into the flanks of $\mathrm{BALB} / \mathrm{c}$ or $\mathrm{C} 57 \mathrm{BL} / 6$ mice, respectively. Tumor growth was measured with calipers every 5 days. On day 21 after inoculation, mice were sacrificed by cervical dislocation.

Cryoslicing and staining for confocal and total internal reflection fluorescence (TIRF) imaging. Subcutaneous tumors were dissected free of the skin and body tissue from Balb/c and C57BL/6 mice. In addition, lung and colon pieces were obtained from healthy nontumor bearing mice. Tumor and normal tissue samples were embedded in Tissue-Tek O.C.T. Compound (Sakura Finetek, Torrance, CA, USA) medium and immediately frozen at $-80^{\circ} \mathrm{C}$. Using a CM 1100 cryostat (Leica, Wetzlar, Germany) frozen samples were cut into serial $10 \mu \mathrm{m}$-thick sections. The cryoslices were placed on $0.17 \mathrm{~mm}$ coverslips suitable for inverted microscopy. The cryoslices were fixed in $4 \%$ PFA for $10 \mathrm{~min}$ at room temperature and washed three times with PBS. In the case of SiR-actin staining, the samples were then blocked for $30 \mathrm{~min}$ in $1 \%$ bovine serum albumin (BSA) in PBS. A mixture of $2 \mu \mathrm{M} \mathrm{SiR-actin}$ (Spirochrome, Stein am Rhein, Switzerland) and Hoechst $33342(2$ $\mu \mathrm{g} / \mathrm{ml}$ ) (Thermo Fisher Scientific, Waltham, MA, USA) in 1\% BSAPBS was used for F-actin and nuclei staining for 1 hour.

Confocal laser scanning microscopy. Imaging of actin cytoskeleton structure in tumor cryoslices was performed on an LSM Meta 510 confocal microscope (Carl Zeiss, Oberkochen, Germany) equipped with Plan-Apochromat $\times 100 / 1.40$ Oil DIC objective. SiR-actinstained microfilaments were imaged under continuous $633 \mathrm{~nm}$ laser irradiation, fluorescence was collected and filtered through BP 650710 IR emission channel. Additionally, femtosecond laser at $800 \mathrm{~nm}$ was used for two-photon excitation of Hoechst 33342, fluorescence emission was recorded at $415-533 \mathrm{~nm}$. Images at 1,024×1,024 pixels were taken both for nuclei and actin visualization.

TIRF imaging. Fluorescent-labelled cells and tissue samples were observed under an Eclipse Ti microscope (Nikon, Tokyo, Japan) equipped with N-STORM system, NIS-Elements Software 4.13.01 and EM-CCD camera iXon3 DU-897 (Andor, Belfast, UK), or the Neo sCMOS camera (Andor, Belfast, UK). The fluorescent images were captured using C-NSTORM QUAD filter cube and $\times 100$ oilimmersion objective Apo TIRF/1.49 (Nikon) in TIRF mode coupled with perfect focus system. We used $405 \mathrm{~nm}$ and $647 \mathrm{~nm}$ continuous lasers, at a range of different powers, for Hoechst 33342 and SiRactin excitation, respectively. Samples were imaged at exposure time of 16-100 ms, EM gain of 200 and conversion gain of 5.1×. A series of sequential images was obtained using "ND Acquisition" control. The size of one camera pixel was equal to $160 \mathrm{~nm}(107 \mathrm{~nm}$ for panel C of Figure 4). For super-resolution imaging of SiR-actin, the samples were imaged under oxygen-scavenging buffer, comprising $\beta$-mercaptoethanol (BME), 14.3 M undiluted stock solution; glucose oxidase from Aspergillus niger, weighed before being added to the buffer; catalase from bovine liver, prepared as a solution in $50 \mathrm{mM}$ potassium phosphate buffer, $\mathrm{pH} 7.0$ (SigmaAldrich, St. Louis, MO, USA). The final imaging buffer contained $10 \%$ (w/v) glucose, GLOX solution $(0.5 \mathrm{mg} / \mathrm{ml}$ glucose oxidase and $40 \mu \mathrm{g} / \mathrm{ml}$ catalase) with $143 \mathrm{mM}$ BME in TN buffer (50 mM Tris, $10 \mathrm{mM} \mathrm{NaCl}, \mathrm{pH} 8.0$ ).

In the case of live-cell imaging, we used the following buffer modification: $2 \%(\mathrm{w} / \mathrm{v})$ glucose, $75 \mathrm{mM}$ Hepes in DMEM with GLOX solution and $10 \mathrm{mM}$ mercaptoethylamine (MEA). We acquired 3,000-10,000 sequential frames for further processing.

Data analysis. Fiji (24) platform was used for all imaging postprocessing steps, including super-resolved image reconstruction. For super-resolution image reconstruction, individual localizations were fitted with ThunderSTORM (25). The GDSC SMLM plugin (Genome Damage and Stability Center, University of Sussex, UK; code available at GitHub: https://github.com/aherbert/GDSCSMLM) was used for Fourier ring correlation analysis. A running median filter was applied to remove slowly changing background 

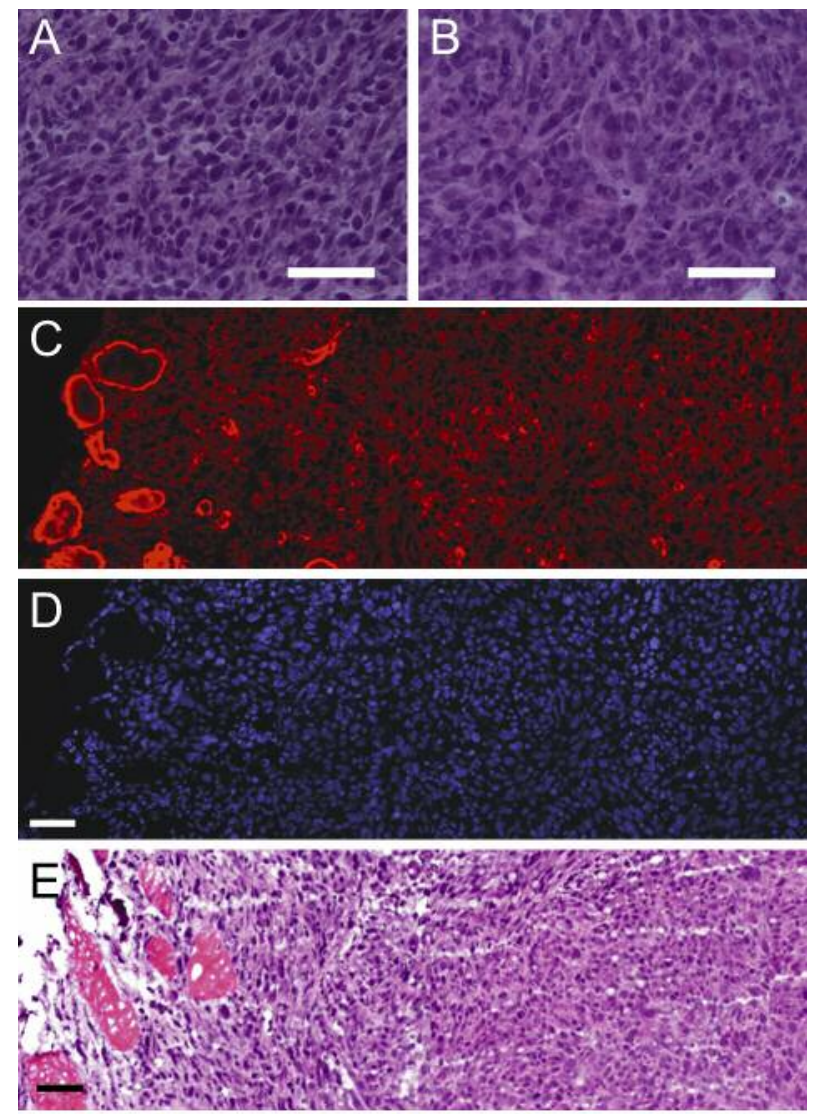

Figure 1. 10- $\mu m$ Cryoslices of CT26 (A) and LLC1 (B) tumor tissues stained with hematoxylin and eosin $(H \& E)$, demonstrating morphologically heterogeneous cell populations. Validation of fluorescence observations against histology: SiR-actin $(C)$, Hoechst $33342(D)$ and $H \& E(E)$ staining of the same region of LLC1 tumor cryoslice. Scale bars $=50 \mu \mathrm{m}$.

(26). A combination of bleaching/blinking assisted localization microscopy (27) and generalized single molecule high resolution imaging with photobleaching (28) type processing (image series subtracting, averaging, and interleaving) was applied for densely labelled parts of the image prior to the localization step. The variance 3D filter plugin for Fiji with kernel size 1-5 in Zdimension was applied for variance-enhanced images.

Histology. Tumor tissue cryoslices of $10 \mu \mathrm{m}$ thickness were fixed in 4\% PFA for $24 \mathrm{~h}$ and stained with hematoxylin and eosin (H\&E) according to standard procedure. Slides were then examined by a qualified pathologist using a Leica DM 1000 microscope with CCD camera DFC290 (Leica) at 100-, 200- and 400-fold magnifications.

\section{Results and Discussion}

We aimed to develop a protocol of intracellular actin patterns visualization in tumor tissues using recently introduced fluorogenic actin-binding small-molecule dye SiR-actin (23).
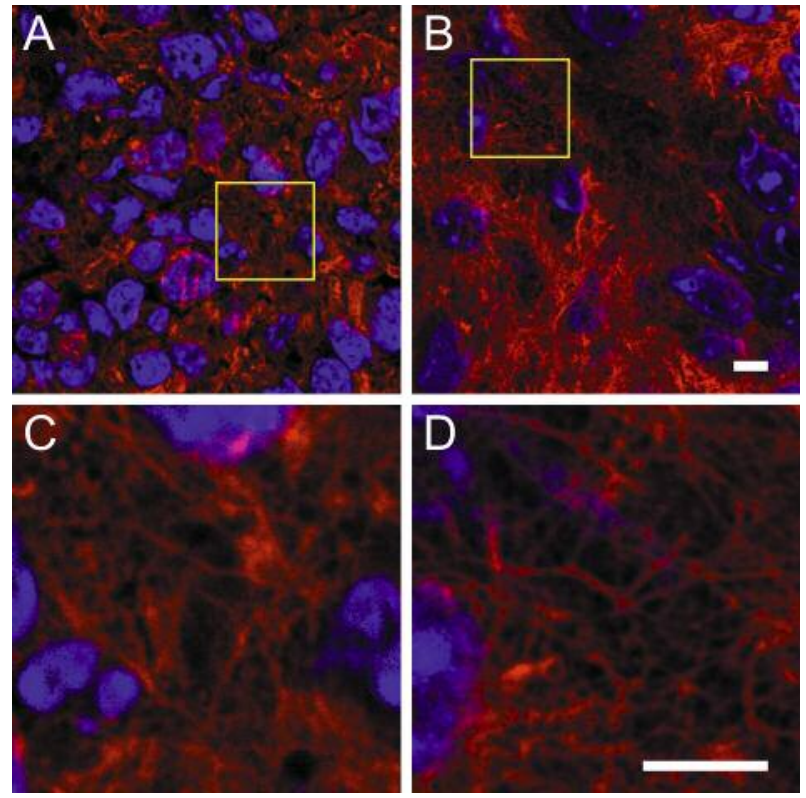

Figure 2. Actin staining in tumor tissue samples. Two-color xy-plane confocal images of tumor cryoslices labelled with SiR-actin (red) and Hoechst 33342 (blue) are shown for CT26 (A) and LLC1 (B) tumor. C and D: Magnified regions from panel $A$ and $B$, respectively. Scale bars $=5 \mu \mathrm{m}$.

Unlike commonly used phalloidin, SiR-actin does not require an extensive washing procedure and allows for labelling of both live and fixed samples. The reduced background owing to fluorogenic properties of SiR-actin should also be beneficial for staining of thick samples. Previously, applications of SiR-actin for super-resolution microscopy were limited to stimulated emission depletion microscopy and structured illumination microscopy (23). We hypothesized that the blinking of carboxyl derivative of Si-rhodamine present in SiR-actin dye could be induced under certain imaging conditions. We tested different buffer compositions and illumination settings in a model of HeLa cells stained with SiR-actin. Samples were imaged in PBS supplemented with BME, as well as in oxygen-scavenging buffer with either BME or MEA. The blinking of SiR-actin was observed only in the case of combination of oxygen-scavenging system and BME or MEA at high illumination intensities $\left(\sim 240 \mathrm{~W} / \mathrm{cm}^{2}\right.$ for $640 \mathrm{~nm}$ laser). It should be noted that SiR-actin blinking was also observed in live cells under these conditions.

We then established the mouse models of CT26 and LLC1 primary tumors. As tumor nodules became palpable and grew progressively, tumor cryoslices were prepared. We examined the cryoslices by histological analysis to confirm that frozen tissue samples consisted of cancer cells. H\&E staining showed a typical tumor pattern both in CT26 and 

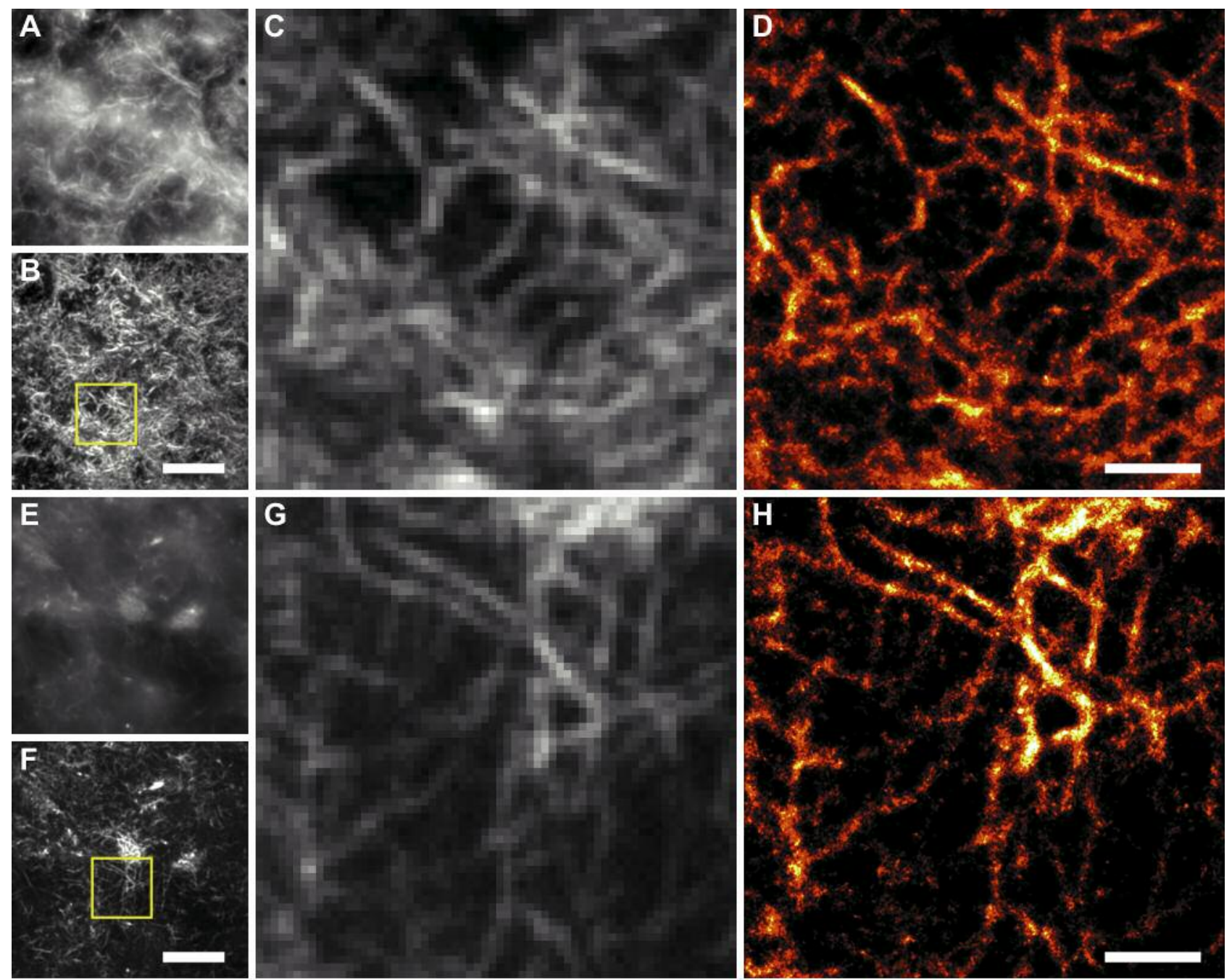

Figure 3. Super-resolution fluorescence microscopy of CT26 (A-D) and LLC1 (E-H) tumor cryoslices stained with SiR-actin. A, E: Shallow-angle images. $B, F$ : Variance-enhanced images (average of running variance computed over 3,000 frames with window size equal to five frames) of panels $A$ and $E$. Scale bars $=10 \mu \mathrm{m} . \mathrm{C}, \mathrm{G}$ : Enlarged regions of areas shown in panels B and F. D, H: Super-resolved images of same regions. Scale bars=2 $\mu \mathrm{m}$.

LLC1 models (Figure 1). Analyzed CT26 samples exhibited dense tissue formed by cells of different size. The cells possessed polymorphic features such as large, round to oval uniform nuclei with diffuse chromatin and one or two nucleoli. The nuclei were surrounded by a relatively thin rim of basophilic cytoplasm. In addition, multiple mitotic phenotypes were detected. In LLC1 tissue, cell morphology was also heterogeneous. Cell shape and size varied greatly. The nuclei were characterized by large multiple nucleoli and extensive regions of condensed chromatin. The tissue had a high proliferative activity as multiple mitoses occurred in the field of view. We also detected some areas of necrosis.

In order to visualize the actin cytoskeleton structure, we performed two-color confocal laser scanning imaging of actin and nuclei in CT26 and LLC1 tumors at high magnification. Surprisingly, a rich network of thick, curved actin bundles was clearly visible within CT26 and LLC1 cancer cells in tumor tissues (Figure 2).

The unexpected finding of thick actin bundles galvanized us to visualize these structures in more detail. We combined shallow-angle (near-TIRF) illumination of $10 \mu \mathrm{m}$-thick tumor tissue slices with SiR-actin blinking to reduce the background and improve image resolution in a non-confocal setup. To induce SiR-actin blinking, the samples were placed in oxygen-scavenging buffer supplied with BME and subjected to high-power red laser illumination. The rapid fluctuations in the fluorescent signal of blinking SiR-actin in the foreground were then separated from the slowly changing 

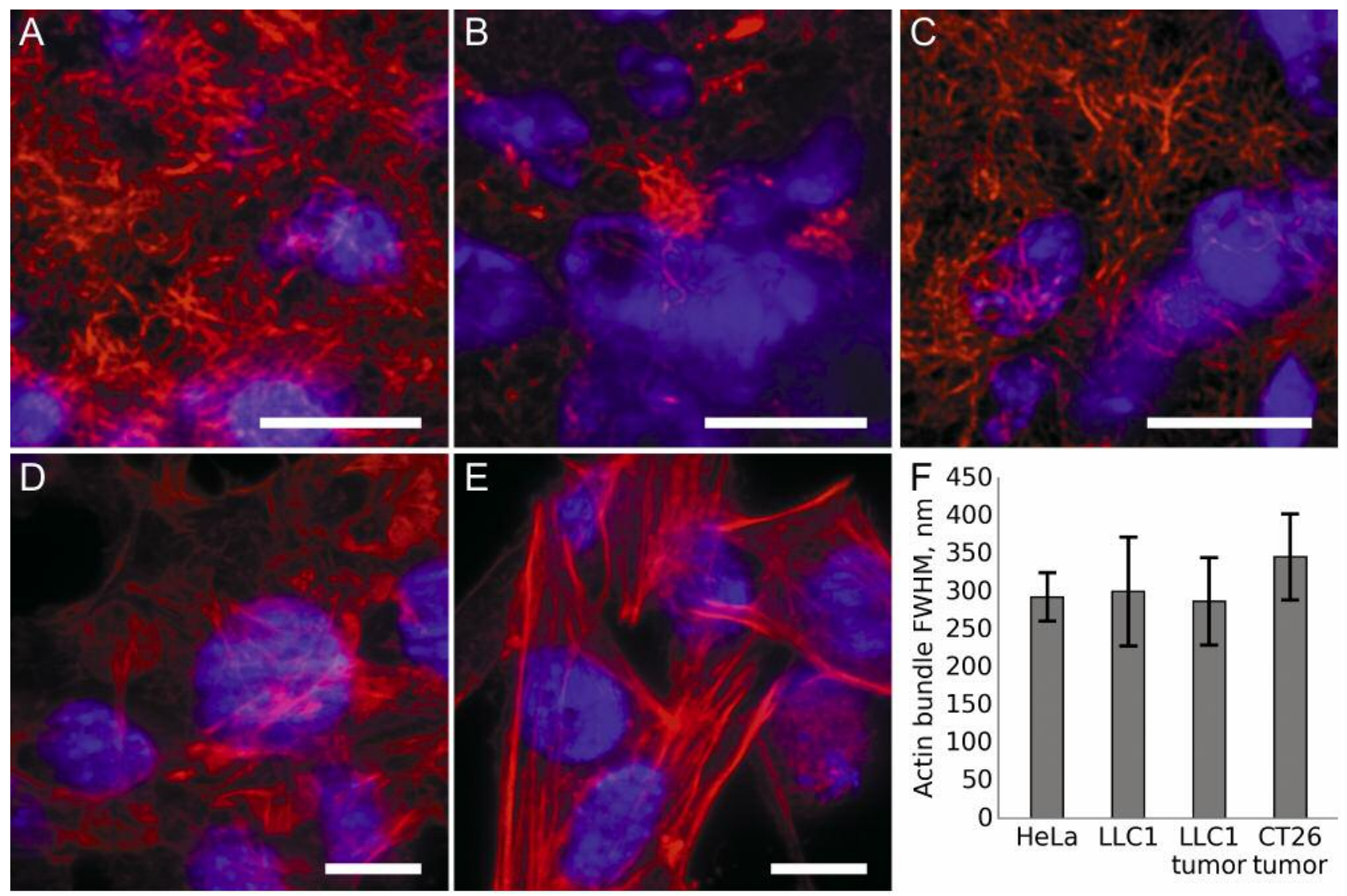

Figure 4. Comparison of actin skeleton in tissue samples of animal tumor models and cell cultures. Samples are stained with SiR-actin (red) and Hoechst 33342 (blue). Tissue samples, red channel - variance-enhanced images (average of running variance computed over 3,000 frames with window size equal to five frames). Fixed CT26 (A) and LLC1 (B) tumor cryoslices, corresponding to regions in Figure $3 B$ and F. C: Non-fixed LLC1 tumor cryoslice. Live CT26 (D) and LLC1 (E) cells in vitro. Scale bars=10 $\mu \mathrm{m}$. F: Half-widths of actin bundles in designated samples. Mean $\pm S D$ values are shown. Each bar represents 60 measurements from three samples.

background signal, similarly to super-resolution techniques based on analysis of fluorescence fluctuations $(29,30)$. Figure $3 \mathrm{~B}$ and $\mathrm{F}$ show a drastic enhancement of the signal from blinking foreground structures by computing the averaged variance of the time series (30). In agreement with the images acquired by confocal microscopy, the network of thick actin bundles was visible in the tumor cryoslices.

In order to determine the thickness of the bundles, we further applied algorithms specifically designed to extract single-molecule localizations from densely labeled samples, and obtained super-resolved images (Figure 3D and H). The signal arising from SiR-actin blinking events perfectly in focus was sufficient to reconstruct super-resolution images with the 50-nm resolution, as determined by Fourier ring correlation analysis (not shown). The thickness of the bundles was found to be similar in all studied samples, including typical straight stress fibers in the cell culture model (Figure 4).
We then performed imaging of normal colon and lung tissue samples with the same staining. Colon and alveolar epithelium of healthy mice were chosen for control experiments with CT26 and LLC1 tumor models, correspondingly. In contrast to tumors, no actin bundles were visible in normal tissues (Figure 5). In the case of colonic tissue, we were able to visualize densely labelled actin, mostly located at the cell apex that was surmised to be actinfilled brush border microvilli. In lung tissues, we observed diffuse microfilaments within alveolar epithelium.

Therefore, actin organization in tumors bore no resemblance to that observed in normal tissues of close origin. Epithelia are very specialized tissues containing cells with strong apical-basal polarity and high adhesive force between each other. The classical model of colonic epithelium revealed by electron microscopy suggests that actin filaments are concentrated in microvilli and almost absent from the rest of the cytoplasm $(31,32)$. Regarding 

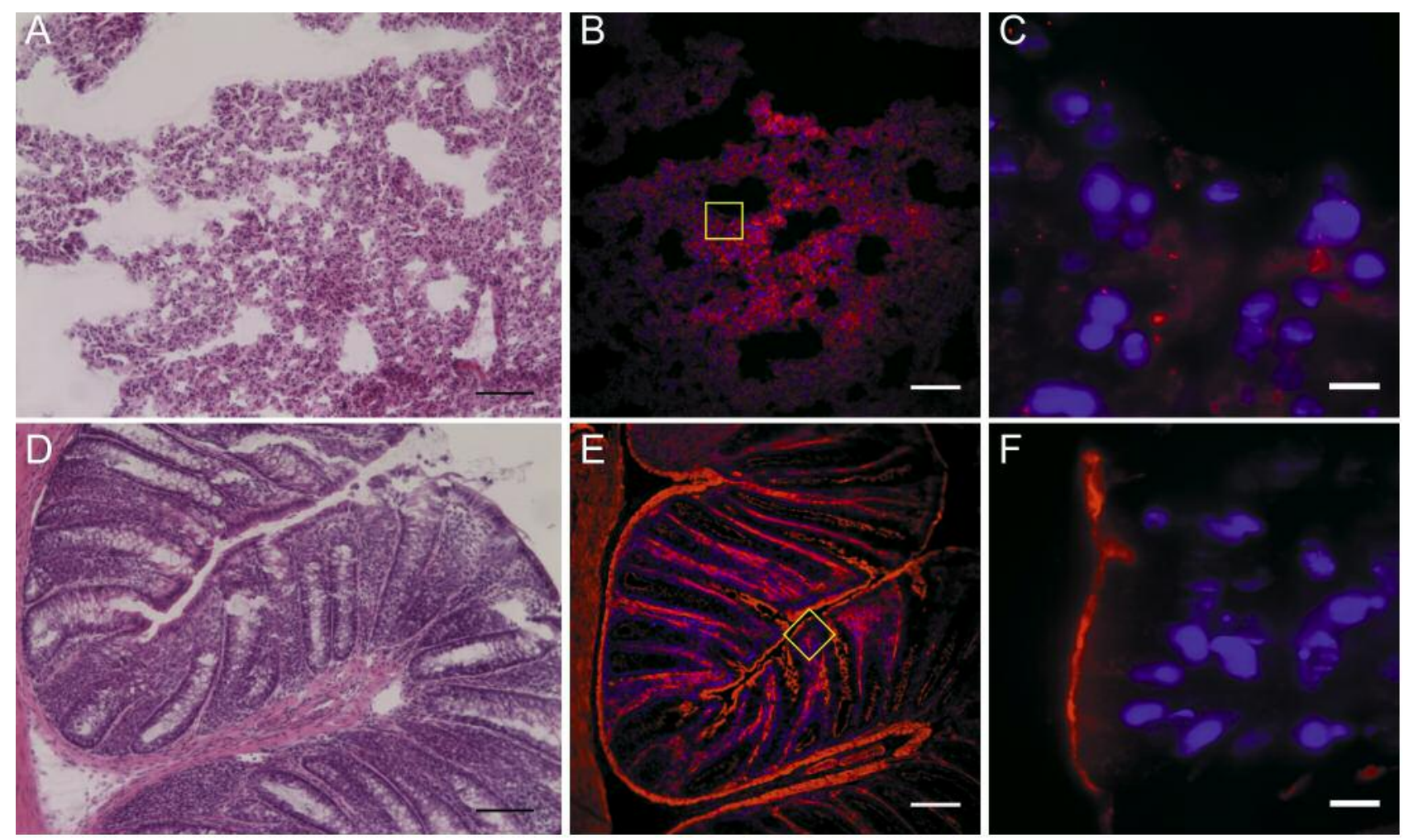

Figure 5. 10- $\mu \mathrm{m}$ Cryoslices of normal lung $(A-C)$ and colonic $(D-F)$ tissues. A, D: Hematoxylin and eosin staining of tissue samples, showing histology of healthy lung and colon. B, E: Total internal reflection fluorescence images of 10- $\mu \mathrm{m}$ cryoslices of healthy lung and colonic tissue, stained with SiR-actin (red) and Hoechst 33342 (blue). C, F: Magnified regions from panels B and E, respectively, marked as yellow squares, showing diffuse microfilaments in alveolar epithelium $(C)$ and actin-rich microvilli $(F)$. Scale bars: A, B, D, E: $100 \mu \mathrm{m} ; C, F: 10 \mu \mathrm{m}$.

alveolar epithelium, actin is poorly and diffusely distributed throughout the cell (33). These results are in agreement with the idea that normal tissue usually lacks stress fibers and their formation strongly depends on environmental conditions, which may be more favorable in tumor (34).

It is widely accepted that stress fibers built of actin and myosin II filaments are widespread in a majority of eukaryotic cells (35). This view arose almost a century ago, however, there has been a much-debated question of whether stress fibers are nothing more than artificial structures. Researchers have revealed that in a particular tissues, cells might develop stress fiber-like structures to withstand high-pressure environments or to increase cell-substrate adhesion (36-38). Nevertheless, the issue whether highly organized actin bundles exist in native tissues or appear only in monolayer cultures on rigid substrata, has not been fully explored.

In this work, we demonstrated that the actin skeleton in cancer cells in vitro significantly differs from that in vivo. In particular, for both CT26 and LLC1 models, long straight stress fibers were observed only in $2 \mathrm{D}$ cell cultures, while an omnidirectional actin mesh composed of thick, curved bundles was visible in tumor tissues. Interestingly, the mean thickness of actin bundles was approximately the same for cancer cells in vitro and in tumors (both fixed and non-fixed samples, Figure 4F). This suggests that the same type of bundled actin structures manifests itself differently in a mechanically strained state in cells on glass slides and in a more relaxed state within tumor-embedded cells. Our data are also in accordance with observations that the formation of tissue-like structures only appears in cancer cells in a 3D matrix mimicking the in vivo environment $(39,40)$.

Summing up, as far as we are aware, for the first time a detailed visualization of the actin cytoskeleton was achieved in tissue samples of different animal tumor models by fluorescence microscopy. In particular, we have revealed that unusual stress fiber-like structures are present within tumor tissue in contrast to cancer cell cultures and normal tissues. We performed super-resolution single-molecule localization imaging of thick tissue samples with fluorogenic probe SiRactin. Although other rhodamine-based dyes have been recently reported for localization microscopy $(41,42)$, there is a need for additional effort to tag actin structures, whereas 
SiR-actin is easy to work with. The experimental approach proposed in this work can probably be directly applied to the study of fresh human cancer specimens, potentially improving diagnostic accuracy.

\section{Conflicts of Interest}

The Authors declare no competing interests in regard to this study.

\section{Acknowledgements}

This work was supported by the Russian Science Foundation grant 14-25-00129. The work was partially carried out using equipment provided by the Institute of Bioorganic Chemistry Core Facility (CKP IBCH).

\section{References}

1 Bezanilla M, Gladfelter AS, Kovar DR and Lee W-L: Cytoskeletal dynamics: a view from the membrane. J Cell Biol 209: 329-337, 2015.

2 Mullins RD, Dyche Mullins R and Hansen SD: In vitro studies of actin filament and network dynamics. Curr Opin Cell Biol 25: 6-13, 2013.

3 McNiven MA: Breaking away: matrix remodeling from the leading edge. Trends Cell Biol 23: 16-21, 2013.

4 Ridley AJ: Life at the leading edge. Cell 145: 1012-1022, 2011.

5 Dominguez R and Holmes KC: Actin structure and function. Annu Rev Biophys 40: 169-186, 2011.

6 Stevenson RP, Veltman D and Machesky LM: Actin-bundling proteins in cancer progression at a glance. J Cell Sci 125: 10731079, 2012.

7 Yamaguchi H, Hideki Y and John C: Regulation of the actin cytoskeleton in cancer cell migration and invasion. Biochim Biophys Acta Mol Cell Res 1773: 642-652, 2007.

8 Pawlak G, Geraldine P and Helfman DM: Cytoskeletal changes in cell transformation and tumorigenesis. Curr Opin Genet Dev 11: 41-47, 2001.

9 Dugina V, Khromova N, Rybko V, Blizniukov O, Shagieva G, Chaponnier C, Kopnin B and Kopnin P: Tumor promotion by $\gamma$ and suppression by $\beta$ non-muscle actin isoforms. Oncotarget 6 : 14556-14571, 2015.

10 Swaminathan V, Mythreye K, O’Brien ET, Berchuck A, Blobe GC and Superfine R: Mechanical stiffness grades metastatic potential in patient tumor cells and in cancer cell lines. Cancer Res 71: 5075-5080, 2011.

11 Pachenari M, Seyedpour SM, Janmaleki M, Babazadeh Shayan $\mathrm{S}$, Taranejoo S and Hosseinkhani H: Mechanical properties of cancer cytoskeleton depend on actin filaments to microtubules content: investigating different grades of colon cancer cell lines. J Biomech 47: 373-379, 2014.

12 Lamouille S, Samy L, Jian X and Rik D: Molecular mechanisms of epithelial-mesenchymal transition. Nat Rev Mol Cell Biol 15: 178-196, 2014.

13 Yuan X, Yu L, Li J, Xie G, Rong T, Zhang L, Chen J, Meng Q, Irving AT, Wang D, Williams ED, Liu J-P, Sadler AJ, Williams BRG, Shen L and Xu D: ATF3 suppresses metastasis of bladder cancer by regulating gelsolin-mediated remodeling of the actin cytoskeleton. Cancer Res 73: 3625-3637, 2013.
14 McKayed KK and Simpson JC: Actin in action: imaging approaches to study cytoskeleton structure and function. Cells 2: 715-731, 2013.

15 Fornasiero EF and Opazo F: Super-resolution imaging for cell biologists: concepts, applications, current challenges and developments. Bioessays 37: 436-451, 2015.

16 Nienhaus K and Nienhaus GU: Where Do We Stand with SuperResolution Optical Microscopy? J Mol Biol 428: 308-322, 2016.

17 Sydor AM, Czymmek KJ, Puchner EM and Mennella V: Superresolution microscopy: from single molecules to supramolecular assemblies. Trends Cell Biol 25: 730-748, 2015.

18 van den Dries K, Schwartz SL, Byars J, Meddens MBM, Bolomini-Vittori M, Lidke DS, Figdor CG, Lidke KA and Cambi A: Dual-color superresolution microscopy reveals nanoscale organization of mechanosensory podosomes. Mol Biol Cell 24: 2112-2123, 2013.

19 Olson MF and Erik S: The actin cytoskeleton in cancer cell motility. Clin Exp Metastasis 26: 273-287, 2008.

$20 \mathrm{Xu} \mathrm{K}$, Zhong G and Zhuang X: Actin, Spectrin, and Associated Proteins Form a Periodic Cytoskeletal Structure in Axons. Science 339: 452-456, 2013.

21 Gatesman Ammer A, Hayes KE, Martin KH, Zhang L, Spirou GA and Weed SA: Multi-photon imaging of tumor cell invasion in an orthotopic mouse model of oral squamous cell carcinoma. $\mathrm{J}$ Vis Exp, 2011.

22 Tsai M-R, Shieh D-B, Lou P-J, Lin C-F and Sun C-K: Characterization of oral squamous cell carcinoma based on higher-harmonic generation microscopy. J Biophotonics 5: 415424, 2012.

23 Lukinavičius G, Reymond L, D’Este E, Masharina A, Göttfert F, Ta H, Güther A, Fournier M, Rizzo S, Waldmann H, Blaukopf C, Sommer C, Gerlich DW, Arndt H-D, Hell SW and Johnsson K: Fluorogenic probes for live-cell imaging of the cytoskeleton. Nat Methods 11: 731-733, 2014.

24 Schindelin J, Arganda-Carreras I, Frise E, Kaynig V, Longair M, Pietzsch T, Preibisch S, Rueden C, Saalfeld S, Schmid B, Tinevez J-Y, White DJ, Hartenstein V, Eliceiri K, Tomancak P and Cardona A: Fiji: an open-source platform for biologicalimage analysis. Nat Methods 9: 676-682, 2012.

25 Ovesný M, Křížek P, Borkovec J, Svindrych Z and Hagen GM: ThunderSTORM: a comprehensive ImageJ plug-in for PALM and STORM data analysis and super-resolution imaging. Bioinformatics 30: 2389-2390, 2014.

26 Hoogendoorn E, Crosby KC, Leyton-Puig D, Breedijk RMP, Jalink K, Gadella TWJ and Postma M: The fidelity of stochastic single-molecule super-resolution reconstructions critically depends upon robust background estimation. Sci Rep 4: 3854, 2014.

27 Burnette DT, Sengupta P, Dai Y, Lippincott-Schwartz J and Kachar B: Bleaching/blinking assisted localization microscopy for superresolution imaging using standard fluorescent molecules. Proc Natl Acad Sci USA 108: 21081-21086, 2011.

28 Simonson PD, Rothenberg E and Selvin PR: Single-moleculebased super-resolution images in the presence of multiple fluorophores. Nano Lett 11: 5090-5096, 2011.

29 Dertinger T, Colyer R, Iyer G, Weiss S and Enderlein J: Fast, background-free, 3D super-resolution optical fluctuation imaging (SOFI). Proc Natl Acad Sci USA 106: 22287-22292, 2009.

30 Watanabe TM, Fukui S, Jin T, Fujii F and Yanagida T: Real-time nanoscopy by using blinking enhanced quantum dots. Biophys $\mathrm{J}$ 99: L50-2, 2010. 
31 Mooseker MS and Tilney LG: Organization of an actin filamentmembrane complex. Filament polarity and membrane attachment in the microvilli of intestinal epithelial cells. J Cell Biol 67: 725743,1975 .

32 Fath KR, Mamajiwalla SN and Burgess DR: The cytoskeleton in development of epithelial cell polarity. J Cell Sci 1993: 6573, 1993.

33 Sormunen R, Pääkkö P, Palovuori R, Soini Y and Lehto VP: Fodrin and actin in the normal, metaplastic, and dysplastic respiratory epithelium and in lung carcinoma. Am J Respir Cell Mol Biol 11: 75-84, 1994.

34 Burridge $\mathrm{K}$ and Wittchen ES: The tension mounts: stress fibers as force-generating mechanotransducers. J Cell Biol 200: 9-19, 2013.

35 Vallenius T: Actin stress fibre subtypes in mesenchymalmigrating cells. Open Biol 3: 130001, 2013.

36 Byers HR, White GE and Fujiwara K: Organization and function of stress fibers in cells in vitro and in situ. A review. Cell Muscle Motil 5: 83-137, 1984.

37 Sugimoto K, Fujii S, Ichikawa Y and Nakamura I: Expression of stress fibers in bullfrog mesothelial cells in situ. Cell Tissue Res 258, 1989.

38 Herman IM, Pollard TD and Wong AJ: Contractile proteins in endothelial cells. Ann N Y Acad Sci 401: 50-60, 1982.
39 Tome Y, Uehara F, Mii S, Yano S, Zhang L, Sugimoto N, Maehara H, Bouvet M, Tsuchiya H, Kanaya F and Hoffman RM: 3 -dimensional tissue is formed from cancer cells in vitro on Gelfoam $^{\circledR}$, but not on Matrigel ${ }^{\mathrm{TM}}$. J Cell Biochem 115: 13621367, 2014

40 Freeman AE and Hoffman RM: In vivo-like growth of human tumors in vitro. Proc Natl Acad Sci USA 83: 2694-2698, 1986.

41 Grimm JB, Klein T, Kopek BG, Shtengel G, Hess HF, Sauer M and Lavis LD: Synthesis of a far-red photoactivatable siliconcontaining rhodamine for super-resolution microscopy. Angew Chem Int Ed Engl 55: 1723-1727, 2016.

42 Uno S-N, Kamiya M, Yoshihara T, Sugawara K, Okabe K, Tarhan MC, Fujita H, Funatsu T, Okada Y, Tobita S and Urano Y: A spontaneously blinking fluorophore based on intramolecular spirocyclization for live-cell super-resolution imaging. Nat Chem 6: 681-689, 2014.

Received August 5, 2016

Revised August 31, 2016

Accepted September 5, 2016 